\title{
HF oder UHF - Welche Frequenz darf es sein? Vor- und Nachteile der gängigen RFID-Technologien
}

\author{
Markus Weinländer, Dieter Horst
}

Die Frage nach der Funkfrequenz eines Radio-Frequency-Identification-Systems (RFIDSystems) ist in etwa wie die Frage nach der PS-Zahl eines Autos: Eigentlich keine primäre Kennzahl für den Anwender wie Höchstgeschwindigkeit oder Beschleunigungsverhalten, aber aufgrund ihrer technischen Bedeutung trotzdem maßgeblich. Ähnlich verhält es sich bei RFID: Zwar stehen eigentlich Eckdaten wie Reichweite, Speicherkapazität oder auch Kosten im Vordergrund. Doch die verwendete Frequenz ist so bedeutend für die Eigenschaften eines RFID-Systems, dass man auch als reiner Anwender kaum an der Frage »HF oder UHF?« vorbei kommt.

\section{Aufbau von RFID-Systemen}

RFID-Systeme bestehen aus drei Hauptkomponenten: einem Transponder mit integrierter Antenne, einem Schreib-/Lesegerät (»Reader«) und einer zugehörigen Sende- und Empfangsantenne. Die Transponder sind Datenträger mit mehr oder weniger Speicherkapazität, die über Funkwellen gelesen und auch beschrieben werden. Häufig sind die Transponder nicht nur lesbar, sondern können auch beliebig oft umprogrammiert (beschrieben) werden. Eine weitere Unterscheidung bezieht sich auf die Energieversorgung: Meist entnehmen die Transponder die gesamte benötigte Energie aus dem elektrischen Feld des Schreib-/Lesegeräts und können deshalb auf eine Batterie verzichten (passiv). Für höhere Reichweiten oder Speicherkapazitäten kann es hingegen notwendig sein, eine Stützbatterie für die Speicherbausteine und die Verarbeitungslogik, nicht aber für die Datenkommunikation einzusetzen (semi-aktiv). Schließlich gibt es auch aktive Transponder, die die eigene Batterie auch zum Senden verwenden; solche Komponenten kommen meist nur in Spezialanwendungen zum Einsatz.

Das Lesegerät samt der zugehörigen Antenne bezeichnet man als Erfassungsstelle. Während die Antenne nur die Funksignale aussendet und empfängt, bildet das Lesegerät eine Art Funkgerät, d. h. die Signale auf der Luftschnittstelle werden in digitale Informationen übersetzt und über geeignete Schnittstellen (z. B. Ethernet) an überlagerte SoftwareSysteme übergeben. Das Lesegerät liefert über die Antenne die benötigte elektrische Energie für den Betrieb der (passiven) RFID-Transponder; gleichzeitig erfolgt über diese sog. Luftschnittstelle auch die Datenkommunikation nach definierten Protokollen. Die praktische Ausführung einer RFID-Erfassungsstelle kann sehr unterschiedlich ausfallen: von kleinen Lesegeräten mit integrierter Antenne bis zu sog. Portalreadern, die mehrere Antennen einsetzen. 


\section{Kriterien für RFID-Systeme}

Aus Anwendersicht wäre, wie eingangs geschildert, die Frequenz eigentlich unwichtig, würde sie nicht in erheblichem Maß die technischen Eigenschaften bestimmen. Wesentliche Parameter für konkrete Anwendungen sind vielmehr der maximale Lese-/ Schreibabstand (Reichweite), die mögliche Speichergröße sowie weitere Eigenschaften wie die Pulkfähigkeit oder unterstützte Standards.

Der gewünschte Lese-/Schreibabstand der Transponder zu den Erfassungsantennen kann zwischen wenigen Zentimetern bis zu mehreren Metern reichen. Allerdings ist hier Vorsicht geboten, denn bei reichweitenstarken Geräten kann es auch rasch zu Überreichweiten kommen: Hier werden Transponder erfasst, die eigentlich gar nicht ausgelesen werden sollen. Die Folgen sind fatal: Wenn z. B. bei einer Anwendung zur Zutrittskontrolle auch andere, weiter entfernte RFID-Chips erfasst würden, so könnte sich jemand unbefugten Zutritt verschaffen.

Sowohl die Frequenz als auch die Antennenkonstruktion bestimmen die erzielbare, maximale Reichweite. Es sollte immer diejenige Kombination aus Frequenz und Antenne gewählt werden, deren resultierender Reichweitenbereich zwar gut für den Anwendungszweck ausreicht, aber nicht deutlich darüber hinausgeht.

Abhängig von Anwendungszweck ist auch die benötigte Speicherkapazität des Transponders ein Kriterium. Ausschlaggebend ist hier das gewählte Datenmodell. Anwendungen nach dem »data-on-network «-Prinzip legen alle ein Objekt betreffenden Informationen in einer (online zugängigen) Datenbank ab und nutzen den RFID-Transponder ausschließlich zur Identifikation. Hier genügt deshalb eine geringe Speicherkapazität von z. B. 96 Bit zur Speicherung einer weltweit eineindeutigen Nummer. Allerdings hat diese Nummer ohne Datenbank-Zugriff keine Bedeutung, was dezentrale Architekturen erschwert. Demgegenüber werden bei »data-on-tag « alle benötigten Informationen direkt auf dem Transponder abgelegt; die Daten sind dadurch dezentral verfügbar, d. h. auch bei Ausfall einer etwaigen zentralen Infrastruktur. Doch die benötigten Speicherkapazitäten können schnell das auf dem Markt Verfügbare übersteigen. Zudem muss ein Konzept überlegt werden, was bei einem Defekt des Datenträgers (z. B. durch mechanische Beschädigung) und dem damit einhergehenden Datenverlust geschehen soll. Für die Praxis empfiehlt sich deshalb eine Mischung beider Konzepte: Einerseits werden die wichtigsten Daten direkt auf dem Transponder mitgeliefert, andererseits aber auch in einer Datenbank gespeichert und um weitere Informationen ergänzt.

Weitere technische Möglichkeiten - entscheidend für die Auswahl der RFID-Produkte - resultieren aus dem Zusammenwirken der Komponenten als Gesamtsystem. Am wichtigsten ist hier die sog. Pulkfähigkeit, d. h. die Möglichkeit, mehrere Transponder gleichzeitig zu erfassen. Sie ist auch ein wichtiger Vorteil von RFID gegenüber Barcodes, weil die Codes nicht vereinzelt werden müssen. So kann z. B. bei einem Wareneingang eine eingehende Sendung in einem Arbeitsschritt komplett registriert werden. Weitere Funktionen betreffen die Datensicherheit der Transponder, wenn z. B. Passwörter oder höherwertige kryptographische Verfahren zum Schutz der gespeicherten Informationen 
eingesetzt werden. Auch die Ortung von Transpondern kann eine gewünschte Eigenschaft sein (wenngleich diese in der Regel nur über spezielle Systeme erreicht wird).

Schließlich spielt der Preis eine wichtige Rolle, vor allem bei Anwendungen, die eine sehr große Menge an Transpondern benötigen (z. B. Logistik). Smart Labels sind einfache Transponder, die wie ein Papier- oder Folienetikett auf einen Artikel aufgeklebt werden. Die Kosten sind gering, dafür sind diese Transponder nicht besonders widerstandsfähig. RFID-Transponder, die dauerhaft eingesetzt werden (z. B. Benutzerausweise), sollten deshalb über ein ausreichend robustes Gehäuse verfügen, das die Funktion auch über mehrere Einsatzjahre sicherstellt - auch wenn die Transponder teuerer sind.

\section{Der Frequenzdschungel}

In Deutschland wurde der Wert von Funkfrequenzen im Jahr 2000 schlagartig deutlich, als die Auktion der UMTS-Lizenzen über 50 Milliarden Euro in die Staatskasse spülte. Funkfrequenzen sind ein rares Gut, weil zahllose Anwendungen um die Nutzungsrechte konkurrieren: von Polizeifunk über Radio und Fernsehen bis zu Babyphone und Funkfernbedienung für die Auto-Zentralverriegelung.

RFID-Systeme können deshalb nicht beliebig auf eine optimale Frequenz und Kanalbreite setzen, sondern müssen das nutzen, was von den staatlichen Regulierungsbehörden zugeteilt wird. Praktisch ergibt das eine Vielzahl von schmalen Frequenzbändern (Abbildung 1). Im Niederfrequenz (Low Frequency, LF)-Bereich werden $125 \mathrm{KHz}$ genutzt, z. B. zur Tieridentifikation. Größere Bedeutung hat das Hochfrequenz-Spektrum (HF) um 13,56 MHz, das gute Erfassungsraten ermöglicht, gleichzeitig aber einen vergleichweise einfachen Aufbau von Antennen, Transpondern und Lesegeräten zulässt. RFID-Systeme auf HF-Basis sind heute weit verbreitet, weil sie vergleichsweise robust funktionieren und einfach eingesetzt werden können.

Seit einigen Jahren sind auch Ultrahochfrequenzen (UHF) im Einsatz, die in Deutschland das Band 865-868 MHz nutzen. Dieses Frequenzspektrum wurde vor allem durch die Maßnahmen des Handels zur Einführung von RFID bekannt. UHF erlaubt vergleichsweise große Reichweiten bei preisgünstigen Datenträgern. Schließlich gibt es auch Geräte im Mikrowellenbereich, z. B. bei 2,45 GHz. Da derartige Systeme aber aktive Transponder benötigen, sind sie eher für Spezialanwendungen im Einsatz. 


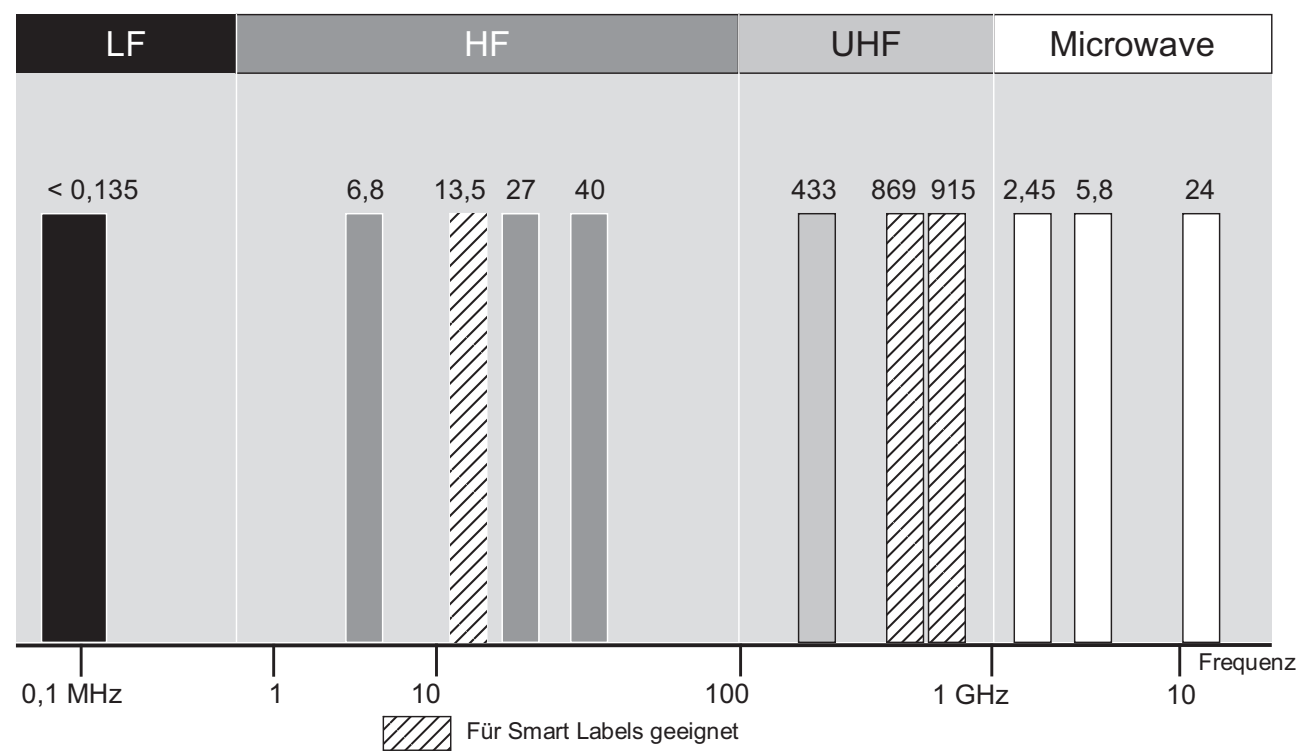

Abb. 1: Für RFID eingesetzte Funkfrequenzen

Damit RFID-Komponenten zueinander kompatibel sind müssen mehrere Voraussetzungen erfüllt sein. Ein RFID-System, das das UHF-Band um $865 \mathrm{MHz}$ nutzt, kann keine Transponder lesen, die für HF ausgelegt sind. Damit nicht genug: Auch das verwendete Datenprotokoll auf der »Luftschnittstelle « muss kompatibel sein. Im UHF-Bereich ist das vergleichsweise einfach, weil sich hier der EPCglobal-Standard »EPC Class 1 Gen2 « durchgesetzt hat und inzwischen auch als ISO-Norm 18000-6C geführt wird. Diese Norm spielt eine erhebliche Rolle bei heutigen und vor allem künftigen RFID-Anwendungen in Logistik und Handel. Die in der Anfangszeit von UHF ebenfalls angebotenen Protokolle ISO 18000-6A und -6B spielen hier kaum noch eine Rolle.

Im HF-Bereich gibt es zwei dominante Standards. Die ISO-Norm 14443 ist vor allem im Bereich Zugangskontrolle, drahtlose Ausweiskarten und Bezahlsysteme eine gewichtige Rolle. Daneben gilt der Standard ISO 15693/18000-3, der sich hauptsächlich für Identifikationsaufgaben in Industrie und Logistik etabliert hat. Beide Standards nutzen zwar die gleiche Frequenz, sind aber nicht zueinander kompatibel.

\section{Technische Grundlagen}

\section{HF-Systeme $(13,56 \mathrm{MHz})$}

Bei Systemen auf HF-Basis wird eine induktive Kopplung zwischen Transpondern und Antennen realisiert. Beide Komponenten verfügen über Spulen, über die Spannungen wie bei einem Transformator übertragen werden können. Abbildung 2 erläutert das Grundprinzip. Der Sender treibt einen Strom durch die Spule der Antenne (in der Abbildung links) und erzeugt so ein Magnetfeld. Durch Induktion wird auf der Empfängerseite (Transponder, rechts) eine Spannung erzeugt, die zum Betrieb des Transponder-Chips 
genutzt werden kann. Die Datenübertragung erfolgt durch die sog. Lastmodulation: Je nach Information (binäre 0 oder 1 ) wird ein Lastwiderstand aufgeschaltet, der zu einem geringfügigen Spannungseinbruch an der Sendespule führt. Diesen kann das Lesegerät auswerten.

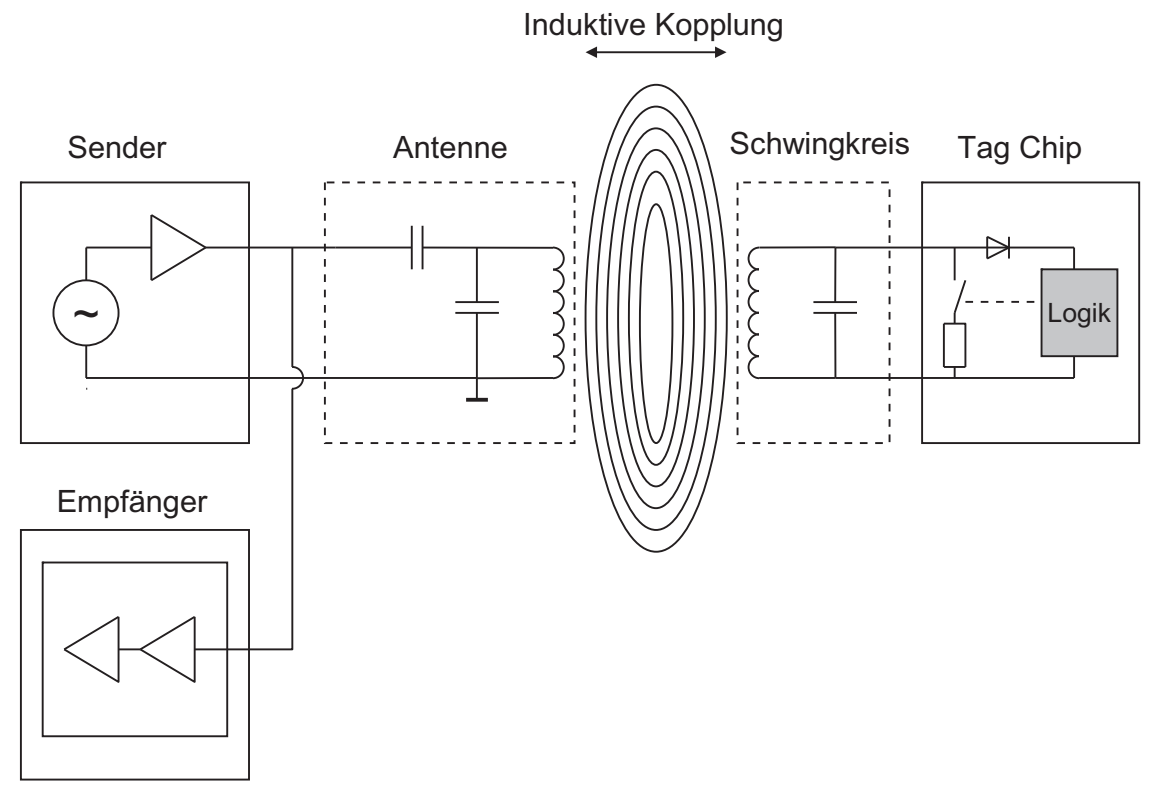

Abb. 2: Aufbau eines HF-RFID-Systems

Durch die Nutzung der induktiven Kopplung bieten HF-RFID-Systeme ein vergleichsweise gut definiertes Übertragungsfeld, das mit zunehmender Entfernung rasch abfällt. Das erfordert zwar auf der einen Seite größere Antennen, wenn größere Reichweiten erzielt werden sollen. Auf der anderen Seite werden durch das begrenzte Lesefeld etwaige Überreichweiten wirksam vermieden. Die charakteristische Spulenform ist bei Transpondern in Smart-Label-Ausführung (d. h. als Etiketten auf Folie oder Papier) gut erkennbar (Abb. 3), wenngleich es die HF-Transponder auch in zahlreichen anderen Bauformen auf dem Markt gibt.

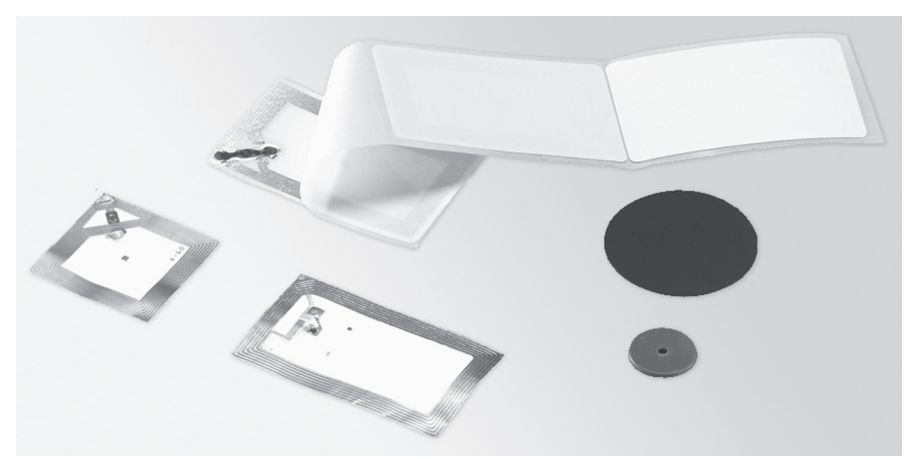

Abb. 3: Typische Bauformen von HF-Transpondern: Links und hinten sog. Smart Labels, rechts Transponder in robustem Gehäuse (Foto: Siemens) 
Die wichtigsten Kenndaten von HF-Systemen sind:

- Reichweite: typisch 50-80 cm, max. 1,5 m (allerdings mit erheblichem Aufwand bei der Antennenkonstruktion)

- Speicherkapazität: 12 Byte - 144 KByte

- Pulkfähigkeit: 60 - 200 Transponder (gemäß ISO 15693)

Bei HF-Systemen sind zudem spezielle Erweiterungen möglich. So nutzen drahtlose Smart Cards, die ein hohes Sicherheitsniveau bieten, meist ebenfalls die Frequenz von 13,56 MHz.

\section{UHF-Systeme (865 MHz)}

Mit dem Aufkommen der RFID-Anwendungen in Logistik und Handel hat sich auch die Nutzung des UHF-Bandes stark erhöht. Allerdings ist die Frequenz nicht weltweit einheitlich nutzbar: das Spektrum reicht von $865 \mathrm{MHz}$ in Europa über $928 \mathrm{MHz}$ in den USA bis hin zu etwa $950 \mathrm{MHz}$ in Japan. Anwender müssen sich darüber jedoch in der Regel keine Gedanken machen, denn die Transponder sind inzwischen so breitbandig ausgelegt, dass sie in allen Regionen gut funktionieren.

UHF-Systeme nutzen eine echte elektromagnetische Kopplung zwischen Transponder und Erfassungsantenne, d. h. neben der magnetischen Feldkomponente wird auch das elektrische Feld genutzt (Abb. 4). Die Sendeleistung von 2 Watt in Europa erlaubt Reichweiten von $5 \mathrm{~m}$ und mehr. Der Transponder verfügt in der Regel über eine mehr oder weniger ausgeprägte Dipolantenne, die die Welle einkoppelt und nach Gleichrichtung dem RFID-Chip zur Verfügung stellt. Die Energieausbeutung ist vergleichsweise gering, so dass nur vergleichsweise einfache Schaltungen mit Strom versorgt werden können dementsprechend klein ist die mögliche Speicherkapazität. Der Chip antwortet durch eine datenabhängige Reflektion des Sendesignals (backscattering).

UHF Lesegerät

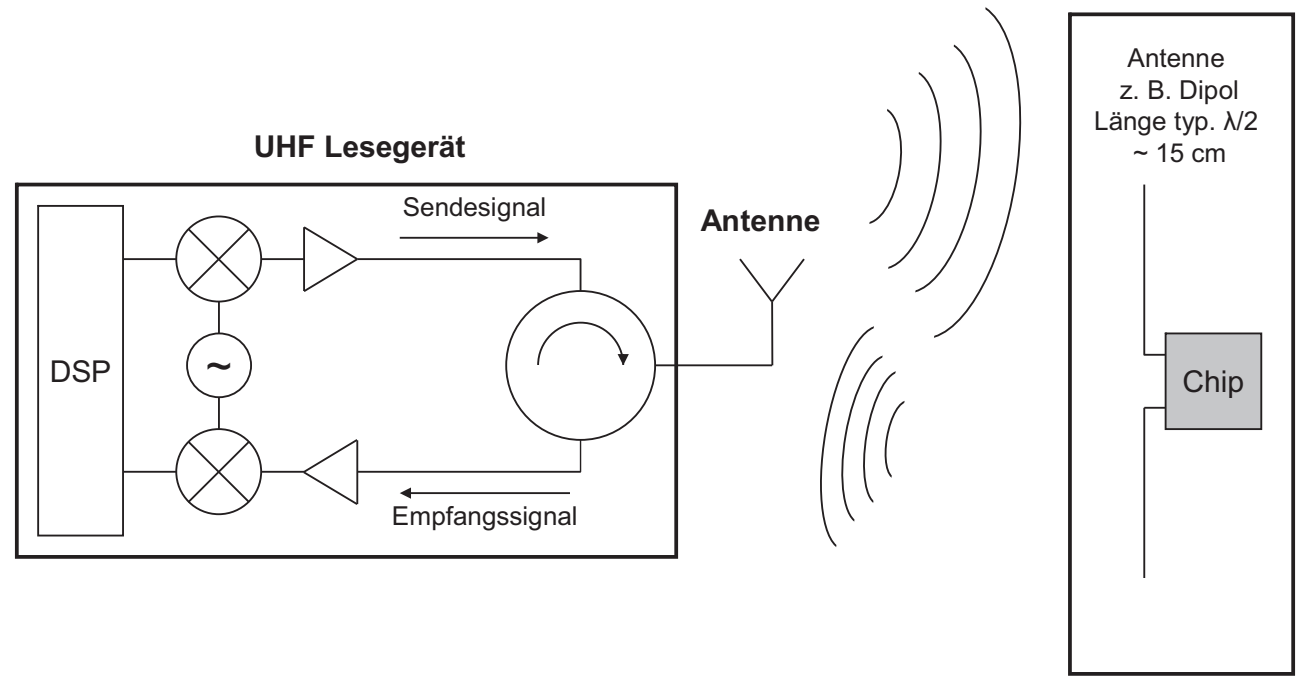

Transponder 
Die Nutzung der elektromagnetischen Kopplung bietet einen großen Vorteil: Die erzielbaren Reichweiten sind erheblich höher als bei HF-Systemen, bei gleichzeitig kleineren Antennen-Abmessungen; typische RFID-Universalantennen für UHF haben Abmessungen um 35 x 35 cm. Die größere Reichweite kann allerdings auch ein großer Nachteil sein, da Überreichweiten oft nicht ohne weitere Maßnahmen (z. B. Abschirmungen, Software-Algorithmen) zu vermeiden sind. Ein weiterer Nachteil ist das durch Reflektionen weniger homogene Feld, das zu »Aussetzern« bei der Kommunikation führen kann. Der Effekt ist ähnlich zum Autoradio, das im Stau plötzlich einen schlechteren Empfang hat als noch wenige Meter vorher. Auch haben Flüssigkeiten und metallische Untergründe einen starken Einfluss auf die Erfassungsqualität (eine Abschirmung verhindert bei HF und UHF gleichermaßen das Auslesen der Chips). Spezielle Transpondertypen eignen sich jedoch für den Einsatz direkt auf Metall.

Allerdings gibt es auch im UHF-Bereich inzwischen Transponder, die überwiegend induktiv (wie bei HF) angesprochen werden. Mit einer auf die magnetische Feldkomponente optimierten Sendeantenne und einem entsprechenden Transponder-Design ist eine Naherfassung ohne Überreichweiten möglich. Werden diese Transponder mit einer sogenannten Hybrid-Antenne ausgestattet, können sie auch weiterhin im Fernbereich über eine »normale« UHF-Antenne gelesen werden.

Die Antennengeometrien bei den UHF-Smart Labels unterscheiden sich von Typ zu Typ ganz erheblich (Abb. 5); je nach Einsatzzweck werden die Transponder dadurch auf spezielle Eigenschaften optimiert (z. B. Größe, Reichweite). Wie bei HF gibt es auch bei UHF Transponder mit robusten Gehäusen für den Dauerbetrieb in zahlreichen Anwendungsszenarien. Einem Transponder mit festem Gehäuse (z. B. laminiert als Scheckkarte) kann deshalb nicht angesehen werden kann, auf welcher Frequenz der Chip operiert.

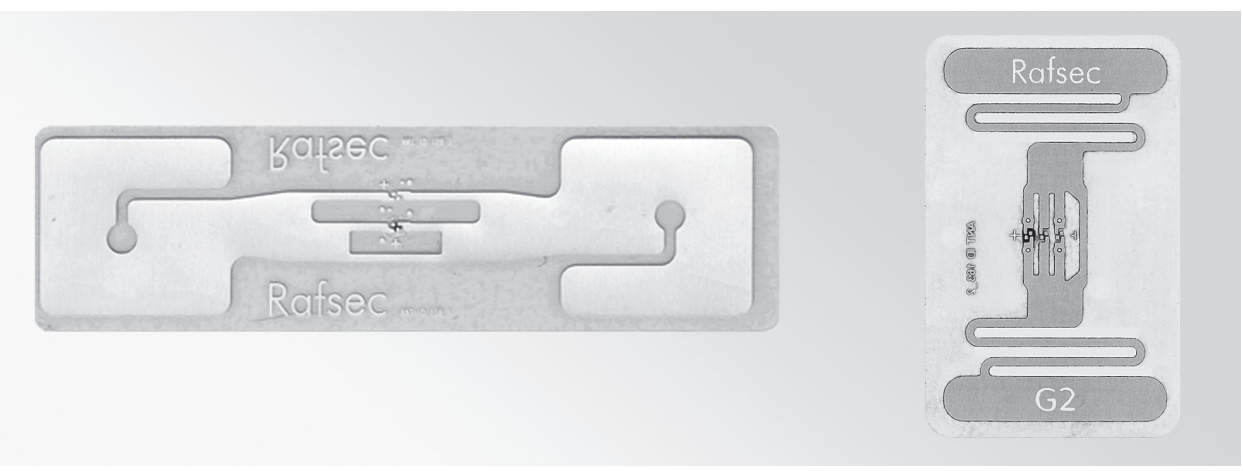

Abb. 5: Beispiele für UHF-Transponder (Foto: Siemens)

Die wichtigsten Kenndaten für UHF-RFID-Systeme lauten:

- Reichweiten bis etwa 5-8 m

- Speicherkapazität: bis 512 Bit

- Pulkfähigkeit: mehrere hundert Transponder

Da aber die UHF-Systeme noch vergleichsweise neu auf dem Markt sind, können für die Zukunft weitere, wichtige Verbesserungen der technischen Daten erwartet werden. 


\section{Welches System ist das Richtige?}

Eine generelle Empfehlung kann kaum ausgesprochen werden, da zu viele Parameter in die Entscheidung einfließen müssen. Auch führt der technische Fortschritt vor allem bei UHF zu einer immer breiten Einsetzbarkeit dieser Systeme. Bei der Auswahl können die folgenden Kriterien hilfreich sein:

- Welche Reichweite muss das System bieten? Wie problematisch erweisen sich Überreichweiten?

- Welche Materialien (Metall, leitende Kunststoffe, Flüssigkeiten) befinden sich im Umfeld?

- Welche Speicherkapazität wird benötigt? Ist »data-on-tag« oder »data-on-network « vorteilhafter?

- Wie viel Platz ist für die Antennen vorgesehen? Wo werden die Antennen montiert?

- Werden spezielle Zusatzeigenschaften gewünscht?

- Welche Standards sind durch die Applikation oder durch mögliche Projektpartner vorgegeben? Was kann sich hier künftig entwickeln?

Aus technischer Sicht sind auf beiden Frequenzen heute Systeme verfügbar, die zuverlässig, robust und mit hoher Leistung ihre Aufgaben erfüllen.

\section{Literatur}

[1] Finkenzeller, K. (2008). RFID-Handbuch. Grundlagen und praktische Anwendungen induktiver Funkanlagen, Transponder und kontaktloser Chipkarten. München: Hanser, 5. Auflage.

[2] Horst, D. (2008). RFID-Technologie. In: Bartneck, N./Klaas, V./Schönherr, H. (Hrsg.): Prozesse optimieren mit RFID und Auto-ID. Grundlagen, Problemlösungen und Anwendungsbeispiele. Erlangen: Publicis, 26-40.

[3] Rankl, W. (2008). Handbuch der Chipkarten. Aufbau - Funktionsweise - Einsatz von Smart Cards. München: Hanser, 5. Auflage. 